\title{
Polydactyly-myopia syndrome
}

INSERM

\section{Source}

INSERM. (1999). Orphanet: an online rare disease and orphan drug data base.

Polydactyly-myopia syndrome. ORPHA:2917

Polydactyly-myopia syndrome is an exceedingly rare autosomal dominant developmental anomaly reported in 1986 in nine individuals among four generations of the same family. The syndrome is characterized clinically by four-limb postaxial polydactyly and progressive myopia. There have been no further descriptions in the literature since 1986. 